\title{
Making it count. An inferentialist account of computer simulation
}

\author{
Jaakko Kuorikoski* \\ University of Tampere, Finland \\ Samuli Reijula \\ University of Tampere, Finland
}

Keywords: inferentialism; scientific representation; computer simulation; similarity

\begin{abstract}
The dual problems of how an idealized model can represent and provide information about its target have become a central topic of in the philosophy of science. We argue that several current views are misguided in assuming that the epistemology of modeling and simulation must build on a philosophical theory of the representation relation (e.g. isomorphism, similarity). We extend Robert Brandom's inferentialist account of meaning into scientific representation to argue that representational language is explicatory, not explanatory, in nature. We provide a broader philosophical rationale for inferential accounts of scientific representation, and an epistemologically modest account of the role of models in terms of inferential scorekeeping. We apply these views to the contested case of computer simulations to argue that, although the praxis of simulation modeling resembles that of scientific experimentation, simulations alone cannot lead to genuinely novel discoveries about the world, as they are merely tools for keeping our reasoning straight.
\end{abstract}

\section{Introduction}

How can highly abstract and idealized models stand for things in the world, and how do they allow us to learn new things about their targets?. These questions seem to arise from a deep philosophical puzzle of scientific representation, which, due to a number of transformations in philosophical discussions, has acquired a central position in the epistemology of science. As the philosophical understanding of science moved from a theory-centered to a modeloriented view of scientific knowledge and practice, questions of approximate truth were reformulated into problems of representation, questions of the logic of inductive inference to ones about model-world inferences andaccounts of explanation by laws to the possibility of explanation via mechanisms virtually isolated in models ([anonymized]). A formidable explanatory burden has thus been placed on a philosophical account of the relationship

\footnotetext{
${ }^{* 1}$ Corresponding author. Prof. Jaakko Kuorikoski, Philosophy / SOC, 33014 Tampere University, Finland.
} 
between our models and the external world. What characterizes theoretical modelling in general is a certain epistemic strategy making use of surrogate reasoning: one first builds something or sets something up, then investigates the properties of that constructed thing, and finally ponders how the discovered properties of the constructed thing relate to the real world (Hughes 1997). This poses an epistemic problem for those empiristically minded - how can we learn something genuinely new about the world without directly empirically investigating the target system itself? Surely the answer to this question has to invoke the fact that the model represents its target. But what if all of this is just wrong?

In this article we argue that this burgeoning philosophical literature on representation is indeed founded on a conceptually questionable basis. We make our case by providing a comprehensive inferentialist account of the representational and epistemic roles of computer simulations. We focus specifically on simulations because the practice of simulation modeling most resembles scientific experimentation in various respects (e.g., Morgan 2005; Mäki 2005; Winsberg 2010; Boge 2018), and it, therefore, provides the most striking case of the problem of accounting for the epistemic added-value of models in general: We seem to learn something genuinely new about the world by manipulating an artificial surrogate system and then "observing" what the end result is. In analytical models, this discovery is a matter of mathematical derivation of a result from the modelling assumptions, but in the case of computer simulations, the analogy between modeling and experimentation feels more compelling. Simulation modeling is therefore the context in which representationalist thinking is most likely to lead into attributing magical epistemology to epistemic artefacts.

Our approach extends Robert Brandom's work to the domain of scientific representation. Inferentialism about model-based representation, broadly defined, states that it is misguided to try to explain the epistemic role of models through concepts such as representation, similarity, or various structure-preserving mappings, since the very relation of representation is constituted by the role of the model in making inferences about the target system. In contrast, we call referentialist accounts all views that start from an account of the representational relation, and then use it to explain (successful) model-world inference. Broadly inferential views have been applied to understanding modelling in general (see Suarez 2004; Contessa 2007; de Donato \& Zamora-Bonilla 2009), but they have not yet been used to account for the representational role of computer simulations. This is not surprising, since the inferential conception is intuitively more compelling in the context of analytical models, whereas computer simulations seem closer to actual experiments, thus including aspects of mimesis, materiality, production of data, and surprise which seem to escape the inferential framework.

We argue that a thoroughly inferentialist view of the 'semantics' of models (a view about the representational content of a model, and about how a model can stand for its target system), also implies a specific view of the epistemological reach of simulations (a view about how and why we can learn something about the target by investigating a model) - a view we call the scorekeeping view. This view denies that models and simulations have the capacity to produce truly new knowledge in the same way as material experiments. It holds instead that their 
epistemic value can be analyzed, without residue, as the improvement of the scope and reliability of our inferences from given empirical premises to empirical conclusions made possible by the model as an external scorekeeping device. Similar views about the epistemology of simulations have been proposed by Otavio Bueno (2014), who argues that the epistemic value of simulations resides in their ability to aid inference making, but then explains this epistemic role in referentialist terms by appealing to structural relations of (partial) homomorphisms. ${ }^{2}$ Claus Beisbart also defends a view about the epistemic reach of simulations similar to ours, but does not take an explicit stance on the semantics of models (2012; 2018, see also Beisbart \& Norton 2012). Nevertheless, he seems to implicitly presuppose some form of intentionalist referentialism, according to which representation is brought about by the intentions of the modeler (2012, p.407), and also links the model underlying the simulation to its intended target in terms of denotation (ibid. p.406).

Our position is in many respects compatible with those of Bueno and Beisbart, but our views on the semantics and the epistemology of simulations rely on a broader outlook on the nature of aboutness or representation in general in deploying Robert Brandom's ideas of deontic scorekeeping and inferential commitments (1994, Ch.3). We therefore complement the inferential theory of scientific representation, originally put forward by Mauricio Suárez (2004), by providing a positive philosophical rationale for the two jointly necessary conditions Suarez proposes for representation, representational force and inference making, and we explain the former in terms of the latter. Together, (inferentialist) inferentialism about representation and the scorekeeping view of the epistemology of modeling show how models and simulations act as inferential crutches in scientific reasoning and argumentation, helping us to keep our reasoning straight. Put bluntly, scientific results are established by arguments. Modeling does not help to establish the empirical premises of such arguments, but they aid with the inferences that take us from those premises to the conclusions, our results.

We take the main advantages of our inferentialist approach to be twofold. First, in relation to simulation models, the approach provides a novel account of the epistemic differences between an artefact being a mere computational device, a computer simulation, and a true experiment. Importantly, our account implies that such epistemic differences are sharp in the sense that they are not blurred either by the fact that the practice of modelling does typically involve an experimental practice, or because evidence from material experiments can often only be applied to scientific reasoning by embedding them as assumptions in models. Our account also has the surprising implication that a physically identical activity (model use) can be epistemically regarded either as modelling or experimentation, depending on the doxastic commitments of the participants. We illustrate our account with an example of computer simulations of crowd dynamics.

Second, in relation to scientific models in general, the inferentialist approach relegates many questions concerning the epistemology of scientific modeling from philosophy to scientific fields in which they can be empirically answered. This is because, according to inferentialism,

\footnotetext{
${ }^{2}$ Bueno also calls his view 'inferential account computer simulation', but we do not use this label here as his account is not primarily about the representational properties of simulations.
} 
representational vocabulary does not have an explanatory, but rather an explicative function: Claims about representation do not pick out relational properties in the world, properties which are somehow responsible for inferential successes, but instead, they simply summarize the inferential activity itself. The task of investigating the successes and failures of these inferential activities is not a philosophical or conceptual one, but an empirical question concerning both the specific subject matter at hand, and the cognitive science of the use of external inferential aids.

Section 2 introduces our approach to representation in general. In section 3 we show how Brandomian inferentialism can be applied to scientific models, and how it leads to a an epistemology of modeling that we call the scorekeeping view. In section 4, we extend the inferentialist treatment to simulation modeling. Section 5 then introduces our example of crowd dynamics and explores the epistemic difference between simulation, computation, and true material experimentation. Section 6 concludes by addressing some of the powerful intuitions motivating the referentialist accounts.

\section{Inferentialism and representation. A primer}

The question of scientific representation is a special case of the more foundational (perhaps the foundational) philosophical question about the nature of aboutness: In virtue of what do some things in the world stand in for other things? This question must be answered whether we are theorizing about the meaning of words, the content of propositional attitudes, or the epistemic role of scientific models.

The distinction between content and use of representations suggests two basic ways in which we can approach the question of aboutness. We can aim to explain what we do with representations (such as understanding the meaning of sentences or reasoning about the world) in terms of the representational content and the representation relation between the representation and the represented thing. This referentialist philosophical programme takes as its points of departure a theory of the representation relation, such as the causal theory of reference (in its many incarnations, see e.g., Fodor 1998), and then explains aspects of the use of representations, such as the systematic and generative nature of language, with the help of this relation. This direction of explanation seems very intuitive: surely it is by virtue of the content of our representations that we are able to do things with them and surely it is by virtue of the objective representation relation that the representations have the content they do.

When trying to understand the representational capacity of scientific models, a proponent of referentialism needs a substantial theory of the representation relation to explain the informational content and the resulting 'epistemic power' of the model. A long tradition in the philosophy of science employed the theory of reference to account for the meaning of theoretical terms and, ultimately, to ground the (approximate) truth of scientific theories (see Stich 1996). But when the focus turned to models, these semantic properties attributable to propositionally structured content felt out of place. Proposed replacements for them include similarity, (partial) isomorphism, and homomorphism. These mappings, so the suggestion 
goes, establish suitably objective relationships between the structure of the representation and the structure of its target, so that they can explain the representational content, and thus the epistemic usefulness, of the model. Also many pragmatic accounts of scientific representation emphasizing the importance of interpretation and thus moving beyond the dyadic understanding of representation are still referentialist in this sense: Although they might not be committed to any specific similarity or structure-preserving mapping, they do rely on the concept of 'denotation', taken as a primitive, for linking the key model parts to the world. Examples of such accounts are R.I.G Hughes' DDI account of representation (Hughes 1997) and the recent DEKI account of model-based representation by Roman Frigg and James Nguyen (2016).

The inferentialist analysis of representation holds that such referentialist direction of explanation puts the cart before the horse. Use cannot be explained by content, which is in turn explained by the representation relation, because content and representation are constituted by use. That is, inferentialism inverses the explanatory roles of content and use. According to inferentialist semantics, the content of linguistic expressions and propositional attitudes are constituted by the inferential commitments undertaken by uttering the expression (Brandom 1994). Roughly, the meaning of a sentence is determined by what other sentences the utterer is now entitled to assent to and to what sentences she is taken to be committed to. For example, if I claim that the temperature of a particular metal rod is 200C, then I'm expected to be able to give reasons concerning measurement processes and observable physical characteristics of the said rod. Some of these commitments have important extra-linguistic aspects, such as the claims concerning acts of possible measurement (subject to language entry-norms) and a practical commitment of not touching it with bare hands (subject to exit-norms). As the popular metaphor has it, grasping the meaning of an utterance is constituted by knowing one's way in the web of reasons, which the utterance is a part of (Sellars 1956).

According to the inferentialist view, full propositional content, genuine aboutness, is possible only within this game of giving and asking for reasons (Brandom 1994). Thermostats and simple organisms react to changes in their environment, but since they cannot relate these actions to other possible actions, the relation between the stimulus and the response is one of simple causation, devoid of any meaning in the full sense. The functioning of the thermostat is caused by temperature, but is not about temperature. Such causal reactions are not correct or incorrect in themselves, they just are. In contrast, meaning and aboutness are normative in that there are correct and incorrect applications of a referring expression.

Here it should be noted that meaning and representation do not directly depend on the intentions of a language user. The commitments and entitlements resulting from speech acts are governed by the principles of language use in the linguistic community, not by the intentions of a single language-user. Furthermore, the game of giving and asking for reasons is an empirically and practically constrained practice, connected to the world through perception and action. (Brandom 1994, 331). Hence, a proponent of inferentialism does not deny the reality or objectivity of representation. There can be facts of the matter about 
assertions (and internalized assertions, i.e. judgments) being correct and mistaken. Furthermore, according to our view, an inferentialist need not be committed to a nonnaturalist ontology of primitive normative facts. She is only committed to an ontology that includes normative acts of sanctioning moves made in the game of giving and asking for reasons. Normativity of meaning is constituted by the other language-users keeping score of what someone said, what follows from it, what is compatible and incompatible with the utterance, and so on.

It is not our aim to argue extensively against the referentialist order of explanation here, although our inferentialism is motivated by a deep skepticism concerning the existence of any substantial representation (or reference) relation, which could explain inferential success (or failure). Instead, we build a positive case for inferentialism by applying the inferentialist approach first to scientific modeling in general, and then to computer simulation in particular. ${ }^{3}$ The resulting analysis of representational notions does not deny the sensibility of referential language, but instead, it helps us to understand what it is that we do when we use the referentialist idiom.

\section{Inferentialist inferentialism about models}

Scientific models are surrogate systems used to make inferences about their targets. This much is agreed on in the philosophical literature on models. Surrogate systems come in many forms and fulfill different inferential functions. Models may be physical scale models, formal models solved analytically with pen and paper, as well as computational models run on a computer. Data models provide systematic and cognitively economic representations of the salient features of data, phenomenological models representations of relevant patterns in the modelled phenomena, and theoretical models aim to represent important features of the causal mechanisms responsible for the modelled phenomenon.

The inferential conception of scientific representation first grew out of the difficulties that similarity- and structural-mapping-based theories had in singling out the correct kind of relation between a model and its target (such as iso- and homomorphisms) (Suarez 2004; Contessa 2007). Such theories which portray representation as a factual two-place relationship between representations and things in the world have difficulties in accounting for the representation relation as non-symmetric, non-transitive and non-reflexive, and struggle in trying to account for the possibility of misrepresentation (Suarez 2003). Furthermore, in light of various modeling practices in the sciences, establishing the sufficiency - or even necessity - of the existence of a particular dyadic relationship for all scientific representation has proved difficult. In their replies to such criticisms, Bueno and French (2011) concede that any dyadic relationship (such as a partial isomorphism) is not alone sufficient for establishing representation in scientific practice - while at the same time insisting on the

\footnotetext{
${ }^{3}$ As far as we know, Brandom does not address in length the question of how scientific theories and models represent. However, see Brandom (1994), pp. 518-520 for his brief remarks on concrete representational devices such as as maps and pictures.
} 
necessity of structure-preserving mappings as the constitutive basis for such practices. ${ }^{4}$ We return to questions of the role of similarity and structure in section 6 . Now, however, we move on to outline how the inferential conception can be developed into a more positive inferentialist account of scientific representation.

According to Suarez's (2004) inferential conception, scientific representation has two necessary conditions: First, a model represents a target only if (1) its representational force points towards it, and (2) the model allows competent and informed agents to draw specific inferences regarding the target. Although the purpose of the second condition is to distinguish scientific representation from mere stipulations or arbitrarily chosen signs, Suarez emphasizes that establishing representation does not imply that the model represents its target completely or even accurately. What makes Suarez's conception a deflationary one is that it does not postulate a deeper (explanatory) property (e.g., similarity or structural mapping) behind the two conditions. Instead, on different occasions, the schema provided by the inferential conception can be filled in in different ways.

The inferentialist nevertheless claims that something more can, in fact, be said: The inferences of condition (2) constitute the representational force of condition (1). Brandom briefly outlines the way in which non-linguistic artefacts can be discursively treated as representations with the familiar example of maps: A map is treated as a representation of some features of the terrain when it is possible to explicitly endorse or criticize inferences from claims about the map to claims about the terrain. The capacity to use something as a representation is therefore, in a sense, parasitic on the aboutness of the claims concerning the map and the terrain, which can in turn be given a fully inferentialist explication (1994, pp.518-519). This narrows down the different ways in which the representational force of an epistemic artefact can be grounded in any given context and removes the need for any further explanation for why precisely these activities are relevant for representation.

As Suarez also points out, representation is therefore an activity and, strictly speaking, any talk of an object being a representation is only a shorthand for talking about its representational potential. The representational potential of a model is constituted by the inferential affordances it provides to the epistemic agents using it to manipulate and navigate the world ([anonymized], [anonymized], Suarez 2004; Vorms 2012). In the context of models, inferring means using formal (syntactic) rules to derive contentful expressions from other contentful expressions in a (within-the-model) truth-preserving way. Such a notion of representational content is a normative one: A model offers an accurate representation of its target in virtue of facilitating correct (outside-the-model) inferences about it. ${ }^{5}$ Unlike in the case of purely linguistic tokens, the correctness of model-based inferences is not ultimately

\footnotetext{
${ }^{4}$ When defending the necessity claim in the section 9.2 of their article, the authors cite Bueno and Colyvan (2011). Bueno and Colyvan, however, acknowledge that they have not established such a result (sec. 6).

${ }^{5}$ By correct we mean inferences resulting in true conclusions. And as with other semantic vocabulary, 'true' is to be understood in an inferentialist manner (as a prosentential operator for taking over the commitments of another expression)Brandom 1994).
} 
determined by social factors (in contrast to the socially-governed use of conventional symbols), but by the empirical success of the inferences (e.g., accurate predictions about the target) and effective language-exit moves (successful actions related to the target) (cf. Brandom 1994, 518-519 on the use of maps) .

Different models afford different kinds of inferences. Descriptive models (such as data models and phenomenological models) facilitate inferences concerning regularities and other occurrent properties of the modelled phenomenon (e.g., statistical inference), and possibly predictive inferences, given that the modelled system is not expected to undergo structural changes. In addition, theoretical models facilitate inferences to counterfactual situations (what-if-things-had-been-different-inferences) and, by virtue of this, provide explanatory information (see Woodward 2003; [anonymized]).

Taking stock, and a as a concrete reference point for the observations below, we suggest the following characterization:

\{IMR\} (Inferentialist analysis of model-based representation): A source object M represents a target system $S$ iff a competent epistemic agent $A$ may use $M$ to make inferences about some subset $P$ of the properties of $S$. $P$ is the set of aspects of $S$ captured by $M$.

The competence requirement is needed on the one hand to rule out inferences based on luck, and, on the other hand, to save the intuition that an object can be said to be a representation, even though only a small group of people can actually use it as a representation (although being a representation is only a shorthand for having the potential to be used in an act of representation). Finally, to say that the source object is only used to make inferences about a specific subset of the properties of the target is to simply acknowledge that all representations are partial.

As with representation in general, the inferentialist denies neither the existence nor the objectivity of model-based representation. For example, causal models represent causal relations in the target system insofar as they facilitate inferences to the consequences of possible interventions on the modelled system. It is just that the representation relation does not explain these inferences. It is constituted by them, or in a less metaphysical idiom, we can legitimately say that a model represents a target because of such inferences. Representational language is a meta-vocabulary which facilitates relating and comparing our differing individual epistemic perspectives on our shared reality. Such vocabulary can be used to summarize which things are - and should be - used to draw inferences about others. Therefore, representational language does not itself "refer" to anything epistemically explanatory (to a "representational hook" anchoring a word to its referent, or a model to its target). The explanatory resources lie elsewhere and are purely empirical: The way in which a cognitive agent can use an artifact to improve the reliability and scope of her inferences concerning another object is explained by (i) the cognitive capacities of the agent, (ii) the causal properties of the artifact and (iii) the properties of the object of interest. There is no additional explanatory challenge to be met by a substantial philosophical theory of the representation relation. 
For example, consider how observing and manipulating an orrery, a clockwork model of the solar system, facilitates inferences about the movement and positions of planets. Here the source (orrery) is a device built in light of our knowledge of the structure of the solar system (target), and physical interaction with the source facilitates inferences about the target.. Conceptually, by drawing conclusions about, for example, the relative positions of the planets at a given time, the model user is making explicit some implications of the modeling assumptions embodied in the model (e.g., relative distances of the planets from the sun). For those implications to be about the target, the modeler must be committed to taking such assumptions as being true of it. The relation between the inferential affordances of the epistemic artifact and the cognitive agent is, however, an empirical object of research for the cognitive sciences, not for philosophy. It is quite natural, then, for the inferentialist to view model-assisted inference as a case of extended (or "scaffolded") cognition, where the use of external tools extends the limited internal cognitive capacities of the agent. ${ }^{6}$

Let us now examine three central implications of the inferentialist view.

(1) Model-based representation is neither subjective nor arbitrary. Although, in principle, anything can be "used" as a model simply by stipulating that object A stands for object B, whether $A$ possesses any such intrinsic features which would enable the model user to improve her inferences about $B$ is certainly not up to the agent to decide. It is also important to note that the mental "intention" of the agent does not by itself, as if by magic, establish the representation relation. The relation holds due to the inferential allowances and commitments that the act of declaring $A$ as a representation of $B$ establishes. For example, it is certainly possible to use a beach ball as a model of the earth, but for most theoretical purposes (above elementary school level), such a model would be epistemically useless: whatever analogies one could find between the coloured stripes of the model object (the source) and the topography of the earth would, in all likelihood, not play a part in any inferences where the examination of the source implies yet unverified properties of the target system. And even when it is possible to specify certain morphisms between the ball and earth, they do not concern properties pertaining to any scientifically relevant aspects of the target.

(2) If the object $M$ systematically leads a competent epistemic agent to make incorrect inferences concerning the set of properties $\mathrm{P}$, then $\mathrm{M}$ misrepresents those properties, and thus partially misrepresents the system $S$ (of course, the agent can also make mistakes in using the model by making wrong within-model inferences, but this is a different kind of error). Like any form of disagreement, misrepresentation is possible only against the backdrop of broader agreement about the use of $M$, i.e., that the inferential use of $M$ is mostly in line with the established doxastic commitments that establish that the use of $\mathrm{M}$ is, nevertheless, about $\mathrm{S}$.

\footnotetext{
${ }^{6}$ We have no interest in taking part in debates on whether such modeling situations should be considered as a case of genuine extended cognition, in contrast to, say, embedded cognition, where the human agent retains a prioritized position (see Clark 2008, Giere 2002 vs. Rupert 2004). Here our aim is only to suggest that, especially from epistemological perspective, the model could be thought of as part of the inference-making system, together with the modeler.
} 
A critic might now think that we are confusing two things: what representations are and what we do with them (Bueno and French 2011). Surely the fact about whether a representation carries information about its target should be prior and independent from whether there are competent users using the representation to produce new knowledge about the target? As stated above, the relevant properties of the artefact, the represented system, and the user all exist independently and prior to the act of representation, but the artefact becomes a representation of its target only in use. What comes to the latter part of the objection, we will next argue that, indeed, (3) models and simulations should not be considered as sources of genuinely new empirical knowledge.

\section{The scorekeeping view of the epistemology of models}

Inferentialism is an account of the 'semantics' of models, i.e. what it means for a model to represent a target.Nevertheless, the denial of any substantial representation relation carrying information from the target to the model also leads to an empiristically plausible view of the epistemic status of models: the scorekeeping view. Since models are, by definition, external inferential aids without any "extra" epistemic relation to their target, they cannot provide any genuinely new empirical information about it (see Giere 2009). The function of models is to improve the scope and reliability of the inferences from empirical assumptions concerning the target system. More specifically, they do this by, quite literally, keeping score of the doxastic commitments and inferential entitlements in our theorization about the world. According to this view, the information learned from the use of models must already be implicit in the modeling assumptions (commitments about the world outside the model):

\{SKE\} (Scorekeeping view of the epistemology of modelling): Models are external doxastic scorekeeping devices. What turns an external artefact, such as a series of equations on a paper or a material scale facsimile, into a model, is that some of its features are treated as "assumptions" about something distinct from and beyond the artefact, the target. By declaring such assumptions, the modeler undertakes doxastic commitments - commitments to treat the consequences of these assumptions as also being applicable, at least prima facie, to the target, and the artefact is constructed in such a way as to facilitate the drawing of further implications of these assumptions.

The function of models is to keep our reasoning straight. Models embody and make explicit doxastic commitments that were previously unavailable to the unaided reasoner and to the epistemic community. By forcing the reasoner to explicitly formalize her assumptions and by facilitating the evaluation of the reasoning, either in terms of correctness of derivation or in terms of sensitivity analysis, models help others to keep score on the commitments undertaken by the modeler. We suggest that ultimately all modeling can be thought of as "extended argumentation." This approach dovetails nicely with many scientists' view of modeling. For example, a textbook on ecological modeling states that "Models do not investigate nature. Instead, they investigate the validity of our own thinking, i.e. whether the logic behind an argument is correct." (Kokko 2007, p.7) In a similar tone, economist Paul Krugman (1998, p.1834) describes the main function of modelling as "keeping things straight" 
and "helping to focus and form intuitions" in contexts involving the adding-up of constraints, indirect chains of causation and feedback effect.

In this sense, models can be compared to thought experiments. Their epistemic output is conditional in nature: both thought experiments and models help us to infer what would follow if certain premises (modeling assumptions) were true. However, unlike thought experiments, modeling forces the theorist to make her assumptions public and explicit, and models are often most useful in making sense of situations where our intuitions are not clear, e.g., where there's feedback, complex chains of causal influence, or several factors pulling into different directions are in play (see Page 2018).

Of course, in all non-trivial cases of model building and use there is psychological novelty and surprise. When the user of an orrery uses the model to predict the relative positions of planets at a future time, these predictions are deductive implications of the implicit commitments concerning the initial conditions and laws, materially realized in the mechanics of the orrery, and undertaken by the modeler when using the artefact as a representation. Although the user can learn new things about the target, because some of the implications of the assumptions used in model construction were initially beyond the reach of her limited unaided inferential abilities, what could be called the extended cognitive system consisting of model together with the model user, learns nothing new (cf. Beisbart 2012, fn. 13). Hence, just as there is no philosophical puzzle of the representation relation, there is no epistemological puzzle of how new empirical knowledge can be gained without direct empirical access to the target.

In sum, models make no epistemic miracles. They can only help to make explicit what was implicit in the modelling assumptions. This applies to analytical models, computer simulations, material simulations and scale-models alike. In contrast, a referentialist explanation of epistemic value in terms of a substantial representation relation between the model and the target is liable to lead to the mistaken idea that information can somehow be conveyed via this special relation from the target to the model (as if by sucking information through a referential straw).

\section{Computer simulation as extended argumentation}

The scorekeeping view downplays the analogy between modelling and experimentation and is most intuitive in the case of analytically solvable theoretical models. Modeling by manipulating a set of equations with pen and paper does not give rise to feelings of empirical exploration and experimentation. Simulation seems like another matter altogether. By simulation we mean the use of the partially autonomous behaviour of a surrogate artefact to mimic some process in the target system (Beisbart 2012; [anonymized]). ${ }^{7}$ The surrogate artefact can be a physical replica (an analogue simulation), such as a model wing in a wind

\footnotetext{
${ }^{7}$ We do not want to enter definitional debates here. We stress the processual nature of simulations so as to rule out purely computational, 'non-mimetic' procedures, such as Monte Carlo methods (see also Hartmann 1996).
} 
tunnel, or a program running on a computer. By partially autonomous we mean that once set up and set running, a simulation run proceeds more or less independently of the intentions and actions of the user. In part, it is this independent and processual behavior of the simulation that makes simulation modeling seem like experimentation.

Here we focus on computer simulations, which also come in several different varieties. It is relatively easy to see how simple toy models like the boid model of flocking behavior by Reynolds (1987), or simple computational realizations of Schelling's (1971) segregation model can be understood to be argumentatively similar to thought experiments. Several assumptions embodied in these models (e.g., about initial positions and movement rules of the individuals) are not directly derived from empirical evidence, but are instead made by the modeler him/herself, and such models are often used to argue for the (im)possibility certain outcomes (Grüne-Yanoff 2009), or to suggest explanatory hypotheses about underlying mechanisms.

However, across the sciences, simulation methods are also used in more data-driven ways. For example, in the social sciences, agent-based models calibrated with large empirical datasets have been used to study why U.S. citizens decide (not to) vote (Rolfe 2012), why crowd disasters sometimes occur at mass events (Moussaid et al. 2011), and how residential and workplace segregation emerges in real cities (Hedström \& Åberg 2005). In addition to explaining phenomena, such models are often used also for the purposes of prediction and policy design (see Bruch \& Atwell 2013). Especially the practice of empirically calibrated agentbased modeling (ECA) resembles empirical experimentation much more than it does analytical modeling (Barberousse et al. 2009; Parker 2009; Winsberg 2009): an artificial replica of the target system is created, it is left to "run", and inferences are then made from the observed results back to the target system. Unlike arguments, the simulation does not output propositional "conclusions", but masses of raw data from which the user must first infer back, by means of further statistical modeling steps, to the properties of the epistemic artefact, and only then to the properties of the simulated system. Due to the rich empirical calibration, such models are also ostensibly 'about' concrete targets, rather than being computational implementations of an abstract underlying model (cf. Bueno 2014).

Claus Beisbart and John Norton have argued that, despite appearances to the contrary, the epistemic role of computer simulations can be fully understood in terms of argument-making: A single run of a simulation is an execution of a series of commands and could thus be thought of as a long deductive argument (Beisbart 2012; Beisbart \& Norton 2012). ${ }^{8}$ Although we agree with Beisbart and Norton's general take on the epistemological role of simulations, this is not our inferentialist notion of an extended argument. In our view, the modeler is not in any meaningful way taking on doxastic commitments that follow from any token execution of a command in a single simulation run. In fact, true deductive "glitches" (to be contrasted to ordinary programming bugs) in such runs are so rare that the modeler would not be held

\footnotetext{
${ }^{8}$ Newell and Simon (1972) noted that a computer program is formally equivalent to a set of difference equations as it determines the new state of the machine as a function of its previous state and new inputs.
} 
epistemically responsible for such clear "malfunctions" of the inferential apparatus. Also, as Beisbart notes, due to roundoff errors, the argument of the modeler is not always the same as that of the algorithm. What the modeler is inferentially committed to is that the implemented algorithm itself does not contain programming errors or add anything substantial to the results and that the results therefore follow only from the stated assumptions. A single run is a concrete causal process and, as such, cannot really be correct or incorrect, and a simulation is not an argument in itself, but a tool for constructing an argument.

In our view, decisions regarding the input parameters and the architecture of a simulation model ought to be conceived as premises in an argument, and salient features of simulation outputs (or interpretations thereof) as its conclusions, the reliability of which is typically assessed by means of sensitivity analysis, i.e. by tracking the changes in outputs resulting from manipulations of the values of the input parameters and the model structure. For example, in empirically calibrated agent-based models, the modeler has to make assumptions about factors such as

[P1] the decision-rules employed by the model agents,

[P2] their form of organization (such as network structure) and interactions,

[P3] initial states and distribution of agent properties and behavioral rules, and

[P4] the structure of the environment. (see Railsback \& Grimm 2019, p.99)

Such assumptions are based on earlier measurements and research, background theory or simply educated guesses. What running the simulation makes possible is the computer-aided tracking - quite literal scorekeeping - of the implications of such assumptions to relevant macro-properties of the model population, including

[C1] its dynamics (equilibria, cycles),

[C2] spatial configurations (e.g., segregation patterns),

[C3] thresholds (e.g., tipping points), and

[C4] diversity effects.

The modeling assumptions regarding $\mathrm{P} 1-\mathrm{P} 4$ are the premises, and the statements about macro-properties [C1-C4] the conclusions of interest of the computationally extended argument. According to IMR, what makes the model a representation is that these premises and conclusions are evaluated with respect to a target system. In agent-based modeling, the premises, taken in isolation, are typically quite simple. The assumed behavioral rules of the agents and their principles of mutual organization are often straightforward and by making such assumptions, the modeler is undertaking conditional commitments that follow from these rules. But due to the local interactions between agents, diversity in their properties, and the sheer size of the simulated population, even after running the simulation, it is often unclear to the model user, how and why a specific set of parameter values results in a particular macro level outcome. 
This inferential opacity of the simulation, i.e., that the sequence of simulation steps is not epistemically accessible to the user (Humphreys 2004), often leads to talk about the emergence of macro properties. We regard such talk as unfortunate and not conducive for understanding the semantics and epistemology of modeling. The word 'emergence' simply labels the point in which our unaided understanding of the dependence between the assumptions and the results ends. There are important questions in the vicinity, however: (1) a semantic question: What determines which parts of the model are really representational? and (2) an epistemic question: How to distinguish genuine results from modeling artifacts? These questions are particularly pressing for simulation models as the inferential connections from premises to conclusions are too complex to be conclusively verified by the unaided model user. This being the case, the relevant inferential system is the model-modeler pair, as suggested above.

From the inferentialist perspective, the semantic and the epistemic problem are closely linked. IMR suggests that a natural starting point for addressing questions about the semantics of the model, i.e. what target systems the model represents and which parts of the model are genuinely representative (in contrast to auxiliary assumptions, programming boilerplate etc.) is to start with the explicitly stated goals of the model users. The statement of modeling goals establishes the doxastic commitments regarding the kinds of things about which the modeler is expected to make inferences, as well provide reasons for these inferences. Hence, the public doxastic commitments determine how the model aims to represent its target. However, as we emphasized above, the question about what the model successfully represents is not up to the modelers intentions. Instead, that depends on the inferential capabilities that the simulation makes possible.

\section{Experiments, analogue models and computer simulations}

With the inferentialist picture of simulations now laid out, let us return to the epistemic differences between genuine experiments and simulations. Among others, Uskali Mäki (2005) and Mary Morgan (2005) have emphasized the analogy between models and experiments in that both involve manipulation of a surrogate object and isolation of a particular (causal) factor of interest. Models are surrogate systems and manipulating them seems to be an indirect way to learn about the target. Furthermore, the practice of modeling can often be described in terms of uncovering a dependency between an independent and a dependent variable of interest. Nevertheless, we have claimed that all modeling, even complex computer simulation, must be understood as extended argumentation explicating the consequences of independently justified empirical premises. We will now apply IMR and SKE in articulating a set of crucial epistemic differences between, on the one hand, computer and analogue simulations, and material experiments, on the other. We do this by examining a case in which equation based models, simulations, as well as material experiments, have been applied to investigate the same event: research on the 2010 Duisburg Love Parade tragedy. We do not use this particular case out of morbid or sensationalist curiosity, but because the phenomenon and its models are theoretically and conceptually easy to understand, thus making the crucial epistemic differences as salient as possible. 


\section{Modeling crowd behavior}

Pedestrian crowds have been studied by scientists for more than half a century now (Hanking and Wright 1958). Henderson (1974) made early theoretical attempts to understand the behavior of crowds by employing differential equations used in fluid dynamics. The notions of flow, pressure, and phase transitions find natural application in the domain of large-scale human crowds (Ball 2006). For example, as crowd density exceeds a critical threshold (approximately 7 people $/ \mathrm{m}^{2}$ ), people have little opportunity to control their movements, and, consequently, shocks and waves get propagated in the crowd in a manner resembling that of a liquid substance.

In addition to such macro models, various kinds of particle, field, and agent-based models have recently been introduced. They aim to augment the understanding of crowd dynamics by describing the crowd at the level of individuals, not of aggregate flows. Such models predict various macro-level phenomena observed in crowds such as lane formation, stop-and-go waves and herding (see Duives et al. 2012). For the current topic, it is notable that both the fluid dynamics model and individual-based models are typically solved by using simulation methods.

Recently, models of pedestrian behavior have been used to explain crowd disasters such as the Love Parade tragedy in 2010. Love Parade was an electronic music festival organized in various German cities since 1989. In the years prior to the 2010 Duisburg festival, the event was yearly attended by more than 1 million visitors. In Duisburg, the event was organized at a former train depot. The area is isolated from its surroundings by railroad tracks on side, and a motorway on the other, and access to and from the festival grounds was through a inverse$T$ shape structure consisting of two long pedestrian tunnels leading to a ramp to the festival area. Only a few hours after the festival opened, severe congestion developed around the ramp as new visitors entered and others were already trying to leave the area due to congestion. In order to control the situation, the police set cordons in both tunnels to keep people from accessing the festival grounds. After a short while, however, the cordons were dissolved. This lead to the development of a two-way flow of people and high crowd densities in the inverse-T structure. During a period of 30-60 minutes, 21 people were killed and several hundred injured due mainly to compression asphyxia (Helbing \& Mukerji 2012; Pretorius et al. 2015).

In the media, escape panic is often stated as the cause of such crowd disasters (Helbing et al. 2000). The mass panic hypothesis suggests that in dense crowds, people start to move faster and interactions become physical in nature. This causes some individuals to fall over and possibly get trampled by the crowd. Furthermore, the behavior spreads in the crowd as people pick up the agitated behavior. Helbing and Mukerji (2012) suggest, however, that it is not clear what role mass panic played in the Duisburg disaster. Instead, based on an extensive analysis of evidence from the festival grounds (e.g. CCTV and participant video), they suggest that the casualties were caused by a phenomenon called crowd turbulence or crowd quake: at dangerously high densities, the crowd becomes almost like a fluid mass, in which shock waves can be propagated over long distances, practically compressing the lungs of the individuals so 
that breathing may become difficult and injured individuals cannot be picked up or transported to safety. However, it also appears that behavioral effects (panic) may explain why the area close to an emergency staircase was the most hazardous one: people trying to make their way to the staircase may have led to the emergence of local pressure spikes in the ramp area.

Pretorius and colleagues (2015) employed an agent-based model to predict the conditions under which crowd quakes and compressive asphyxia could occur. They simulated six alternative scenarios using an ABM calibrated with detailed evidence about visitor arrival and departure rates as well as geographical modeling of the festival grounds. The first simulation of the actual events appeared to satisfactorily reproduce events according to the original timeline. In the five remaining scenarios, the authors manipulated the occurrence of the police cordons, effects of signposting, flow separation and alternative exit and entry strategies. Their results suggest, for example, that were the temporary police cordons not set in place, crowd densities would not have reached dangerously high levels and the catastrophe could have been avoided.

Although the empirically calibrated modeling conducted by Pretorius et al. (2015) does share many of the pragmatic elements of running material experiments, our SKE account of the epistemic function of simulations suggests that even such highly mimetic models are argumentative tools tracking the inferential entitlements of the doxastic commitments encoded in the modeling assumptions. It is just that in the empirically calibrated models, the assumptions include richer information about an actual scenario (e.g. the Duisburg event), whereas theoretical toy models embody mainly general assumptions about the kinds of processes involved in crowd dynamics. Despite appearances to the contrary, the processual and mimetic characteristic of realistic, empirically calibrated simulations do not epistemically set them apart from more abstract kinds of modeling involving no such virtual communities of agents. The epistemic specialty of agent-based models lies elsewhere: unlike analytical modeling, computational modeling allows the combination of a large set of premises - most distinctively assumptions concerning various types of heterogeneity of agent properties.

From the point of view of the modeler, there is a clear aspect of genuine experimental praxis and surprise in working with an ECA. When the modeler manipulates the external epistemic artefact, she, as a boundedly rational agent, can learn genuinely new things about its inferential properties. However, the genuinely novel material inferences involved in such experimentation on the model do not concern the target of the model, but rather the properties of the modelling apparatus itself. So what gets increased here is her understanding of the model, and the modeler's abilities to successfully manipulate the external inferential apparatus in a goal-directed manner. This provides a legitimate indirect epistemic role for "conceptual exploration" in providing better understanding of our inferential tools. Robustness/sensitivity analysis is also an important part of learning about models because it is a way of evaluating and alleviating the unreliability of the inferential apparatus brought about by its inferential opaqueness. 
From the point of view of the extended cognitive system comprised of the model user and the model, however, manipulating a model ${ }^{9}$ is simply an argumentative move, a move in the game of giving and asking for reasons, giving rise to a new inference from the altered assumptions to a new conclusion. Different kinds of inferential roles for agent-based models (elaboration of a possible mechanism, integration of data, prediction, policy-advice) require different kinds of justificatory arguments and different manipulations of the model. But unlike in genuine experiments, these justificatory arguments have to, in the end, rely on separate empirical premises coming from outside of the model (inferential "model entry" moves).

For example, as Moussaid et al. (2016) point out, the fluid dynamics and particle based models cannot provide understanding about the influence of behavioral effects (e.g. panic) on crowd dynamics, as they make no commitments about the decision processes of the individuals. To address this challenge, Moussaid and coauthors collected evidence of human behavior in such situations by running group experiments using virtual reality headsets. Unlike modeling, such experiments can act as sources of discovery about human behavioral effects in dense crowds. Adding modeling assumptions regarding such behavioral effects into a simulation can provide suggestions of the systemic effects of such patterns. Although such effects could not have been foreseen by an unaided human reasoner, the effects were already implicit in the empirically established modeling assumptions.

But why did the experiment carried out in virtual reality provide genuinely new information whereas the agent-based models did not? And are we simply stipulating what is to be argued for, or have we stretched the concept of argumentation so far as to also cover experimentation as some kind of material argumentation? Although experiments and simulations seem to incorporate a similar epistemic dynamic comprising of artefact construction, manipulation and external inference, philosophical analyses of their epistemic differences have been surprisingly varied. The common intuition is that true material experiments are obviously epistemically more powerful than simulations. There is, however, disagreement about where this advantage comes from.

The most obvious difference would seem to be that true experimentation involves direct causal manipulation of the very stuff investigated, and many philosophers have articulated this difference in terms of the material basis of the direct, experiment-based inferences. However, Francesco Guala (2002) acknowledged that the simple intuition of direct manipulation of the physical target is not enough to distinguish between the epistemic roles of experiments and models, because both crucially involve an external validity inference from a source to a separate target. Nevertheless, Guala argues that the difference lies in the "deep" material basis of inferences based on true experiments (see also Morgan 2003). Wendy Parker (2009) has also argued that materiality as such cannot be the thing that makes the difference, because computer simulation studies are also fundamentally material experiments on the computer (see also Barberousse at al. 2009). Margaret Morrison (2009) goes further in denying that there is any fundamental difference, because both experimentation and

\footnotetext{
${ }^{9}$ For example, running the otherwise same ABM (e.g., Schelling model) with the local social interactions between agents constrained first to von Neumann neighborhood and then to Moore neighborhood.
} 
simulation are model-mediated to similar degree and that at least some simulation results can therefore be seen as indirect experimental measurements of the properties of the target system.

We lack the space to argue against these views in detail ${ }^{10}$ and simply build on Eric Winsberg's work (2009), which pinpoints the difference to the fact that the extrapolative arguments from simulation and experiment to their targets are justified differently: arguments from simulations rely on "principles of model building" whereas arguments from experiments rely on material similarity. Although in broad agreement with Winsberg, we now argue that the inferentialist perspective provides a more nuanced articulation of this epistemic difference. As a starting point, let us consider what, if anything, distinguishes material simulations (analogue models) like wind tunnels or physical fluid models of crowd dynamics from computer simulations? Both kinds of model seem to share the experiment-like epistemic dynamic, and although they have a different "material" relation to their targets, they can both be used to serve the same epistemic functions. Consider an anecdote about John von Neumann told by Winsberg (2010). Von Neumann and his research team had been using a physical wind tunnel to study highly intractable problems in fluid dynamics, but the use of the wind tunnel involved various material inconveniences. When the laboratory finally acquired a computer powerful enough to calculate the wanted results based on physical laws, von Neumann expressed relief that they no longer needed the cumbersome physical wind-tunnel to perform those very same calculations. Von Neumann had used the analogue model purely as an inferential aid, a material calculator, without invoking any "same matter" inferences. Although "material", the wind tunnel was therefore not a true experiment. But what made the epistemic difference? It cannot be the intentions of the modeler, because it is not clear why private mental states should make such an epistemic difference. Mere intending does not establish representation.

From the inferentialist perspective, the epistemic difference arises from the prior (public) doxastic commitments undertaken by the modeler(s) and the consequent differences in the justification for distinguishing genuine results from mere artefacts. When an external inferential aid is treated as a simulation, these judgments follow directly from the commitments defining the representational properties of the inferential apparatus, i.e., what parts of the model are "fair-game" for empirical criticism. What it is for an external system to be used as a representation of some target is that the inferences it facilitates are, in principle, based on already articulated commitments about its target. In contrast, if the system is used as a true experiment, then these judgments can also appeal to previously unknown properties of the material basis of the system itself (open-ended "model entry" moves). Although also inferences from experimental systems (laboratory set-ups or samples from a larger populations) to their "targets" are also partly based on commitments about the target, the

\footnotetext{
${ }^{10}$ We agree with Parker that, from the perspective of the modeler, computer simulation can be described as involving experimentation on the computer, as the modeler learns new things about the epistemic artefact used. Nevertheless, from the epistemically important perspective of the modelmodeler combination, simulation modeling is computationally extended argumentation w.r.t. to the modelled target system.
} 
experimental system is a representative, not a representation, of its target. For example, Von Neumann's wind tunnel was a model (a representation) of abstract fluid dynamics, in virtue of his team being committed to only those results that really follow from the principles of fluid dynamics. This makes the analogue model a representation of these abstract principles, not of any concrete physical system. If the materiality of the wind tunnel had caused systematic deviations from the results mathematically implied by the intractable fluid dynamics equations, the results would have appropriately been judged to be artefacts. Thus physically identical modelling situations (model-modeler-target) can be representationally distinct when embedded in different games of giving and asking for reasons.

This underscores our point that representation is a social, even "normative", accomplishment, not a two-place relation in the world. The epistemic difference between true experiments and virtual "experiments" (i.e. models) is neither arbitrary, up to the intentions of any particular individual, nor a matter of an intrinsic material similarity or dissimilarity of the model and the target. The material properties of the virtual experiments are in no direct way responsible for their epistemic role. The epistemic difference is, nevertheless, categorical, and arises from the doxastic commitments undertaken when treating an external inferential apparatus as $a$ representation of some target. These doxastic commitments determine what the model-user can appeal to when drawing conclusions with the aid of the model, as well as the scope of reasons that can be appealed to when criticizing these conclusions (i.e., whether they are warranted or mere artefacts of the inferential apparatus).

\section{7 "In the end, there has to be similarity ..."}

"Similarity, ever ready to solve philosophical problems and overcome obstacles, is a pretender, an impostor, a quack." (Goodman 1972)

"Invoking a relation (for instance some sort of isomorphism) between representing and represented does not by itself contribute to the task of explaining what the intelligibility of the representing consists in" (Brandom 1994, 74)

Having laid out our inferentialist account of models and simulations, we now return to the powerful intuition underlying its alternative the referentialist order of explanation. According to referentialism, there must be an explanation for the inferential success of a model in terms of some kind of similarity or isomorphism relation between the model and the target. The inferentialist does not, of course, deny that successfully representing models are, in some respects, similar to their targets. The problem is with the presupposition that there has to be a substantial fact of the matter about such a similarity that is be independent of our inferential practices in a way that allows it to explain these inferential practices. We do not claim that inferential practices should be taken as primitive brute facts without any explanation, but that such explanations in terms of similarity or isomorphism are vacuous pseudo-explanations.

As critics of referentialist positions have repeatedly argued, there is no such thing as similarity per se. Nelson Goodman famously quipped that everything is similar to everything in some respect, and that the very notion therefore becomes vacuous. Hence, representation must 
involve relevant similarities. As even the defenders of structural-referentialist accounts currently appear to admit, such relevance cannot be determined without reference to our practices (Bueno \& Colyvan 2011; Weisberg 2013). We suggest that the meaning of a similarity claim is explicable by the inferential commitments undertaken by the speaker: "A is similar to $B$ " obliges the utterer to assent or give reasons for claims of the form "If $P(A)$, then $P(B)$ ". If most of these claims (where the properties $\mathrm{P}$ belong to some set taken as relevant in the context) turn out to be false, then the similarity claim is false. Similarity cannot be used as a general explanation, because claims of similarity just state that these inferences can be made - which was just what was in need of an explanation to begin with.

However, this does not mean that judgements of similarity are arbitrary or subjective. We cannot make analogical inferences successful (truth conducive) simply by wishing so. Moreover, the success in each case is explained by the objective material properties of the things compared, and by the (perfectly objective) cognitive make-up of the user of the representation. What the inferentialist inversion does imply, however, is that there is no such thing as naked similarity "out there."

We suspect that not even the more sophisticated versions of the similarity account, such as that of Michael Weisberg (2013), can escape this basic insight. Weisberg develops a measure of similarity in terms of the ratio of shared and unshared features of the compared objects. But this only shifts the problem a step further to the delineation of said features: one cannot count features before they are defined, and the question of defining features is always a question of relevance. And as Weisberg himself notes, such questions are always relative to the community practices of the relevant field. The more consensus there is about what the relevant features are, the less room there is for reasonable disagreement about similarity.

Accounts based on structure-preserving mappings seem to ameliorate the problem of defining relevant similarity. Consider what is perhaps the most straightforward case, isomorphism. Structures are isomorphic purely and objectively in virtue of the relations between their parts. But, again, picking out the structures that concrete objects instantiate is a matter of discerning the relevant from the irrelevant. As proponents of the structural approach (Bueno and Colyvan 2011; Bueno 2014) themselves suggest, this already constitutes an inferential step from the phenomenon to a model ("immersion"). In cases where targets (and sources) are rich in potentially representable structures, understanding how and what a model represents requires shifting attention from the structures themselves to the inferential commitments determining which of the potentially identifiable structures are the relevant ones.

It does not follow from inferentialism that (structural) similarities between model and target could not have a role to play in any particular story about scientific modeling. In fact, we do not find much to disagree with the way that the modeling-related reasoning practices are described by Bueno (2014). The disagreement concerns the epistemic role of an account of the representation relation - whether a conceptual analysis of the relation, formulated in terms of structural similarities and denotation, plays a role in explaining epistemic success. Unlike Bueno, we do not regard claims about representation as explanatory. Even in Bueno's 
own account, in which the epistemic adequacy of a model is described in terms of the model capturing the correct counterfactual patterns of dependency between data inputs and model outputs, it is not obvious that a theory of the representational relation as a partial morphism is an informative part of the explanation of such adequacy. Instead, for the inferentialist, being able to perceive the model and target as instantiating the same abstract structure is a consequence of getting the modeling commitments right. Representational notions are explicative and expressive, not explanatory (Brandom 1994, 330-333).

\section{Conclusions}

We have shown how inferentialism about the semantics of simulation models, combined with the scorekeeping view of their epistemology, result in a satisfying analysis of claims about how and what such virtual experiments represent, and how such claims are related to the epistemic role of models and simulations in scientific practice. Inferentialism suggests that claims about a model (or its components) successfully representing a target are ultimately explicative, not explanatory nor justificatory. Claims about representation summarize our current knowledge of the sphere of reliable model-based inferences concerning the actual and possible targets - they do not refer to epistemically explanatory relationships in need of a philosophical theory. Consequently, the inferentialist perspective raises concerns about the fruitfulness of many aspects of the current philosophical research programme on scientific representation.

The score-keeping view suggests that simulations should be understood as algorithmic elements in arguments produced by the coupled model-modeler system, and as such they do not provide genuinely new empirical knowledge about their targets. Although the praxis of simulation has many elements in common with that of material experimentation, our analysis suggests that the processual and mimetic characteristics of simulations do not have any epistemic added value beyond what can be legitimately understood as argumentative moves from model assumptions to conclusions.

The inferentialist framework could also be used to explicate possible differences between simulations in natural science, especially physics, and social-science simulations. In physics, for example, the greater confidence in background theory, i.e. the theoretical premises, and the accuracy of measured input values can render simulations quite alike model-mediated indirect measurements, as Morrison (2009) suggested. If such confidence greatly exceeds the maximum reliability of any material experiment on the system itself, then the results from virtual experimentation would epistemically trump material experimentation - at least within the scope of the deductive consequences of the underlying theory.

\section{References}

Barberousse, A., Franceschelli, S., \& Imbert, C. 2009. Computer simulations as experiments. Synthese, 169, 557-574.

Beisbart, C. 2012. How can computer simulations produce new knowledge? European Journal for Philosophy of Science 2: 395-434. 
Beisbart, C. 2018. Are Computer Simulations Experiments? And If Not, How Are They Related to Each Other? European Journal for Philosophy of Science 8 (2): 171-204.

Beisbart, C. \& Norton, J. 2012. Why Monte Carlo Simulations Are Inferences and Not Experiments. International Studies in the Philosophy of Science 26 (4): 403-422.

Boge, F. forthcoming. Why computer simulations are not inferences, and in what sense they are experiments. European Journal for Philosophy of Science. https://doi.org/10.1007/s13194-018-0239-z

Brandom, R. 1994. Making it Explicit: Reasoning, representing, and discursive commitment. Cambridge, MA: Harvard University Press.

Bruch, E. \& Atwell, J. 2013. Agent-Based Models in Empirical Social Research, Sociological Methods \& Research. Online version.

Bueno, O. 2014. Computer simulations: an inferential conception, The Monist 97, 378-398.

Bueno, O. \& French, S. (2011). How theories represent. British Journal for the Philosophy of Science, $62(4): 857-894$

Bueno, O., \& Colyvan, M. 2011. An inferential conception of the application of mathematics, Noûs 45, $345-374$.

Bygren, M. 2013. Unpacking the causes of segregation across workplaces, Acta Sociologica 2013, 56, 3-19.

Clark, A. 2008. Supersizing the Mind. Oxford University Press.

de Donato, X., \& Zamora-Bonilla, J. 2009. Credibility, idealisation, and model building: An inferential approach. Erkenntnis 70: 101-118

Fodor, J. 1998. Concepts: Where cognitive science went wrong. Oxford: Clarendon Press.

Frigg, R. \& Nguyen, J. 2016. The Fiction View of Models Reloaded. The Monist 99: 225-242.

Giere, R. 2002. Scientific cognition as distributed cognition. In P. Carruthers, \& S. Stich \& M. Siegal (Eds.), The Cognitive Basis of Science (pp. 285-299). Cambridge: Cambridge University Press.

Giere, R. 2009. Is computer simulation changing the face of experimentation? Philosophical Studies 143: 59-62.

Grüne-Yanoff, T. 2009. Learning from minimal economic models. Erkenntnis 70, 81-99.

Guala, F. 2002. Models, simulations, and experiments. In L. Magnani \& N. Nersessian. (Eds.), Model-based reasoning: Science, technology, values (pp. 59-74). New York: Kluwer. 
Hartmann, S. 1996. The World as a Process: Simulations in the Natural and Social Sciences, in Hegselmann, R., Müller, U., \& Troitzsch, K. (eds.). Modelling and Simulation in the Social Sciences from the Philosophy of Science Point of View. Dordrecht: Kluwer, 77-100.

Hedström, P. \& Åberg, Y. 2005. Quantitative research, agent-based modelling and theories of the social, In Hedström 2005: Dissecting the Social. Cambridge University Press.

Henderson LF. The statistics of crowd fluids. Nature 1971;229:381-3.

Hughes, R. I. 1997. Models and representation. Philosophy of science, 64, S325-S336.

Kokko, H. 2007. Modelling for Field Biologists and Other Interesting People. Cambridge University Press.

Krugman, P. 1998. Two Cheers for Formalism. The Economic Journal 108, 1829-1836.

Morgan, M. 2005. Experiments Versus Models: New Phenomena, Inference and Surprise. Journal of Economic Methodology 12 (2), 317-329.

Morrison, M. 2009. Models, Measurement and Computer Simulation: The Changing Face of Experimentation. Philosophical Studies 143 (1), 33 - 57.

Moussaid, M., Helbing, D., Theraulas, G. 2011. How simple rules determine pedestrian behavior and crowd disasters. PNAS, 108, 6884-6888

Mäki, U. 2005. Models Are Experiments, Experiments Are Models. Journal of Economic Methodology 12 (2), 303-315.

Page, Scott E. 2018. The Model Thinker: What You Need to Know to Make Data Work for You. Basic Books.

Parker, W. 2009. Does matter really matter? Computer simulations, experiments, and materiality. Synthese, 169, 483-496.

Railsback, S. F., \& Grimm, V. 2019. Agent-based and individual-based modeling: a practical introduction. Princeton university press.

Reynolds, 1987. Flocks, herds, and schools: A distributed behavioral model. Computer Graphics 21:4, 25-34.

Rolfe, Meredith. 2012. Voter Turnout. Cambridge University Press.

Rupert, R. 2004. Challenges to the hypothesis of extended cognition. Journal of Philosophy, 389-428.

Schelling, T. 1971. Dynamic models of segregation. Journal of Mathematical Sociology, 1:143186 
Sellars, W. 1956. Empiricism and the Philosophy of Mind, in Minnesota Studies in the Philosophy of Science, vol. I, H. Feigl \& M. Scriven (eds.), Minneapolis, MN: University of Minnesota Press, 1956: 253-329.

Stich, S. 1996. Deconstructing the Mind. New York: Oxford University Press.

Suarez, M. 2003. Scientific representation: against similarity and isomorphism, International Studies in the Philosophy of Science, 17.

Suarez, M. 2004. An Inferential Conception of Scientific Representation, Philosophy of Science, 71, 767-779.

Suarez, M. 2010. Scientific representation. Philosophy Compass 5, 91-101.

Vorms, M. 2012. Models and formats of representation in Humphreys \& Imbert (eds.):

Models, Simulations, Representations. Routledge.

Weisberg, M. 2013. Simulation and Similarity. Oxford University Press

Winsberg, E. 2009. A tale of two methods. Synthese, 169, 575-592.

Woodward, J. 2003. Making Things Happen: A theory of causal explanation. New York: Oxford University Press. 